\title{
Piecewise Conserved Quantities
}

Tevian Dray

\begin{abstract}
We review the treatment of conservation laws in spacetimes that are glued together in various ways, thus adding a boundary term to the usual conservation laws. Several examples of such spacetimes will be described, including the joining of Schwarzschild spacetimes of different masses, and the possibility of joining regions of different signatures. The opportunity will also be taken to explore some of the less obvious properties of Lorentzian vector calculus.
\end{abstract}

\section{Introduction}

In 1987, my wife (Corinne Manogue) and I found ourselves in India for 6 months, where we were both Indo-American Fellows. The highlight of our visit was the 3 months we spent at TIFR, working with Paddy and others in the Theoretical Astrophysics Group. During this visit, Paddy and I wrote a paper on piecewise Killing vectors [17], bringing additional mathematical clarity to the intriguing results I had previously obtained with 't Hooft [9] on shells of matter in Schwarzschild spacetimes. Little did I know at the time that this theme would recur in my research in a variety of contexts (and more than a dozen papers) over the next 20 years.

As I congratulate Paddy on the occasion of this Festschrift, it is with great pleasure that I look back on this period near the beginning of our careers. It seems only fitting that I use this opportunity to summarize my own journey through several quite different applications of piecewise conserved quantities.

In Sections 2 and 3 I lay out the framework for analyzing piecewise structures, then in Section 4 provide the main mathematical result, the Patchwork Divergence Theorem, generalizing my work with Paddy [17]. The two basic applications are considered next, namely shells of matter in Section 5, and signature change in Sec-

Tevian Dray

Department of Mathematics, Oregon State University, Corvallis, OR 97331, USA, e-mail: tevian@math.oregonstate.edu 
tion 6. In Section 7 I point out how the underlying framework of the Patchwork Divergence Theorem also provides insight into vector calculus in Lorentzian geometry, providing access to these ideas for non-experts, including undergraduates. Finally, a very brief summary is given in Section 8

\section{Piecewise Smooth Tensors}

The long history of the distributional curvature due to piecewise smooth metric tensors is summarized in [1]. As discussed there, the basic setup is two smooth manifolds $M^{ \pm}$joined along a (possibly null) hypersurface $\Sigma$, with smooth metric tensors $g_{a b}^{ \pm}$on either side. Introducing a step function $\Theta$ which is 0 on $M^{-}$and 1 on $M^{+}$, the metric on $M=M^{-} \cup M^{+}$is given by

$$
g_{a b}=(1-\Theta) g_{a b}^{-}+\Theta g_{a b}^{+}
$$

One traditionally assumes that the metric is continuous at $\Sigma$,

$$
\left[g_{a b}\right]:=\left.g_{a b}^{+}\right|_{\Sigma}-\left.g_{a b}^{-}\right|_{\Sigma}=0
$$

in which case the connection $\Gamma^{c}$ ab is at worst discontinuous and, as discussed briefly in [9], it is straightforward to compute distributional curvature tensors. For example, the distributional Ricci tensor is given by

$$
\begin{aligned}
R_{a b} & =(1-\Theta) R_{a b}^{-}+\Theta R_{a b}^{+}+\delta_{c}\left[\Gamma^{c}{ }_{a b}\right]-\delta_{b}\left[\Gamma^{c}{ }_{a c}\right] \\
& =(1-\Theta) R_{a b}^{-}+\Theta R_{a b}^{+}+\delta \rho_{a b}
\end{aligned}
$$

where

$$
\delta_{c}=\delta n_{c}=\nabla_{c} \Theta
$$

so that $n_{c}$ is normal to $\Sigma$, and the distribution $\delta$ can be thought of as a Dirac delta function. However, as shown in [1], it is enough for the pullbacks of $g_{a b}^{ \pm}$to agree on $\Sigma$ in order to have a well-defined tangent space on all of $M$, which we will exploit in Section 6

More generally, we can consider other piecewise smooth tensors on $M$, such as a vector field of the form

$$
\xi^{a}=(1-\Theta) \xi_{-}^{a}+\Theta \xi_{+}^{a}
$$

and its discontinuity $\left[\xi^{a}\right]$, defined in analogy with (2).

\section{Piecewise Conserved Quantities}

As is well-known, a Killing vector $\xi^{a}$ can be contracted with the stress-energy tensor $T_{a b}$ to yield a conserved quantity 


$$
X^{a}=T^{a b} \xi_{b}
$$

satisfying

$$
\oint X^{a} N_{a} d S=0
$$

where $S$ is any closed, piecewise smooth hypersurface (assumed for the moment to be nowhere null) with unit normal vector $N^{a}$. The vanishing of this integral is a consequence of the Divergence Theorem, since

$$
\nabla_{a} X^{a}=\nabla_{a}\left(T^{a b} \xi_{b}\right)=\left(\nabla_{a} T^{a b}\right) \xi_{b}+T^{a b} \nabla_{a} \xi_{b}=0
$$

the first term vanishes by energy conservation, and the second by Killing's equation.

As defined in [17], a piecewise Killing vector is a piecewise smooth vector field $\xi^{a}$ on $M$ of the form (5), such that $\xi_{ \pm}^{a}$ are Killing vectors on $M^{ \pm}$. However, piecewise Killing vectors are not in general Killing vectors on $M$, since

$$
\nabla_{(a} \xi_{b)}=\left[\xi_{(a}\right] \delta_{b)}
$$

which is nonzero if $\xi^{a}$ is discontinuous. Referring to (8), we see that

$$
\nabla_{a}\left(T^{a b} \xi_{b}\right)=\left[T^{a b} \xi_{a}\right] \delta_{b}
$$

If $\Sigma$ is a spacelike hypersurface, and if the Darmois junction conditions (continuity of both the intrinsic metric and the extrinsic curvature) are satisfied there, then the stress-energy tensor is continuous at $\Sigma\left(\left[T^{a b}\right]=0\right)$, and 10$]$ reduces to

$$
\nabla_{a}\left(T^{a b} \xi_{b}\right)=T^{a b}\left[\xi_{a}\right] \delta_{b}
$$

A natural condition on $\xi^{a}$ is for its tangential components to agree on $\Sigma$, in which case

$$
\left[\xi_{a}\right]=\Xi n_{a}
$$

for some function $\Xi$ defined on $\Sigma$. Given (12), and using (4), we will obtain a conserved quantity so long as

$$
T^{a b} n_{a} n_{b}=0
$$

on $\Sigma$. If $\Sigma$ is spacelike, (13) asserts that the energy density at $\Sigma$ seen by an observer orthogonal to $\Sigma$ must vanish.

\section{The Patchwork Divergence Theorem}

In the language of differential forms, the divergence of a vector field $X$ is defined in terms of the volume element $\omega$ as

$$
\operatorname{div}(X) \omega:=£_{X} \omega
$$


where $£$ denotes the Lie derivative. Using Stokes’ Theorem in the form

$$
\oint_{\partial W} \alpha=\int_{W} d \alpha
$$

and the identity

$$
£_{X} \alpha=d\left(i_{X} \alpha\right)+i_{X}(d \alpha),
$$

where $i$ denotes the interior product, one obtains the Divergence Theorem in the form

$$
\int_{W} \operatorname{div}(X) \omega=\oint_{S} i_{X} \omega,
$$

where $S=\partial W$. Any 1-form $m$ orthogonal to $S$ determines a unique volume element $\sigma$ on $S$ through the requirement that

$$
m \wedge \sigma=\omega
$$

$\sigma$ is compatible with the induced orientation on $W$ precisely when $m$ is outward pointing. Using the properties of the interior product, the Divergence Theorem becomes

$$
\int_{W} \operatorname{div}(X) \omega=\oint_{S} m(X) \sigma .
$$

So long as $S$ is not null, the right-hand side of 19 ) is the same as the integral in (7) after obvious identifications.

For piecewise smooth tensors, we can apply (19) separately on $M^{ \pm}$and then add the results. Given a region $W=W^{+} \cup W^{-}$overlapping $\Sigma$, we let $S=\partial W$ and $S_{0}=$ $W \cap \Sigma$. We can extend $m$ to outward-pointing 1-forms $m_{ \pm}$orthogonal to $S^{ \pm}=\partial W^{ \pm}$; on $\Sigma$ we have $m_{-}=-m_{+}=: m_{0}$. The Patchwork Divergence Theorem [7] then takes the form

$$
\int_{W} \operatorname{div}(X) \omega=\oint_{S} m(X) \sigma-\int_{S^{0}} m_{0}([X]) \sigma^{0} .
$$

Our convention is that $m_{0}$ is the 1 -form pointing from $M_{-}$to $M_{+}$; which way the physically equivalent vector field points depends on whether $\Sigma$ is spacelike, timelike, or null.

\section{Shells of Matter}

A simple model for matter falling into a black hole consists of spherical shells of massless matter. Remarkably, as shown originally by 't Hooft [9], this situation can be described by an exact solution of the Einstein field equation, at least in the context of piecewise smooth tensors. The special case of a single massless particle sitting at the horizon of a Schwarzschild black hole [8] remains the only explicitly known exact solution in general relativity that describes a test particle moving in the field of another object, and is in this sense the only known solution to the relativistic two- 
body problem. These models have been generalized to charged black holes [11], to colliding shells [10, 9, 2], and, more recently, to shells of negative energy [21].

In [17], we considered two Schwarzschild spacetimes with different masses joined along a null cylinder $\Sigma=\{u=\alpha\}$ representing a spherical shell of massless dust. The corresponding metric is

$$
d s^{2}= \begin{cases}-\frac{32 m^{3}}{r} e^{-r / 2 m} d u d v+r^{2} d \Omega^{2} & (u \leq \alpha) \\ -\frac{32 m^{3}}{r} e^{-r / 2 M} d U d V+r^{2} d \Omega^{2} & (u \geq \alpha)\end{cases}
$$

where $U$ and $V$ are functions (only) of $u$ and $v$, respectively, and

$$
\begin{aligned}
u v & =-\left(\frac{r}{2 m}-1\right) e^{r / 2 m} & & (u \leq \alpha), \\
U V & =-\left(\frac{r}{2 M}-1\right) e^{r / 2 M} & & (u \geq \alpha) .
\end{aligned}
$$

Continuity of the metric requires that on $\Sigma$ we have

$$
\frac{\alpha}{m}=\frac{U(\alpha)}{M U^{\prime}(\alpha)}=: \gamma
$$

which implies that

$$
\frac{u \partial_{u}}{m}=\frac{U \partial_{U}}{M}
$$

on $\Sigma$. The only nonzero component of the stress-energy tensor is

$$
T_{u u}=\frac{\delta}{\gamma \pi r^{2}}(M-m)
$$

and we have the piecewise Killing vector

$$
\xi=(1-\Theta) \frac{v \partial_{v}-u \partial_{u}}{4 m}+\Theta \frac{V \partial_{V}-U \partial_{U}}{4 M} .
$$

Thus, $[\xi]$ is proportional to $\partial_{V}$, satisfying condition (12), while [13) is satisfied by virtue of the double-null form of the stress-energy tensor.

We therefore obtain an integral conservation law of the form (7). Since the support of $T_{a b}$ is on $\Sigma$, we obtain a conserved quantity $Q$ by evaluating this integral over any hypersurface $S$ intersecting $\Sigma$ only once. We choose

$$
S= \begin{cases}\{t=\text { const }\} & (u \leq \alpha) \\ \{T=\text { const }\} & (u \geq \alpha)\end{cases}
$$

where $t$ and $T$ denote Schwarzschild time in the regions $u \leq \alpha$ and $u \geq \alpha$, respectively, and where the constants are chosen so that $\Sigma$ is continuous. Putting this all together, we have

$$
-Q=\int_{S}\left((1-\Theta) T^{t}{ }_{t}+\Theta T^{T}{ }_{T}\right) d S
$$


which appears to involve the distributional product $\delta \Theta$. However, since

$$
\frac{\partial u}{\partial r}=\frac{u}{4 m} \frac{1}{1-r / 2 m}
$$

it turns out that

$$
T^{t}{ }_{t}=T^{T}{ }_{T}=-\frac{\delta\left(r-r_{0}\right)}{4 \pi r^{2}}(M-m)
$$

where $r_{0}$ is the radius of the shell where $S$ intersects $\Sigma$. Thus, there is no actual step function present in the integrand in (29). Finally, evaluating the integral leads to

$$
Q=M-m
$$

which shows that the energy of the shell is precisely the difference of the two Schwarzschild masses, as expected.

Thus, the results of [17] can be regarded as an application of the Patchwork Divergence Theorem in this setting.

\section{Signature Change}

"Spacetimes" combining both Lorentzian and Euclidean regions were proposed independently by George Ellis's group in the context of early universe cosmology [19, 18] and by our group in the context of quantum field theory in curved space [14, 15, 16]. Subsequent work by both groups addressed tensor distributions [4, 20] and the distributional field equations [3, 6], in the process realizing that conservation laws would take a different form at a change of signature [22], ultimately leading to the Patchwork Divergence Theorem [7].

The key point is that, even though the metric is clearly discontinuous at a change of signature, 1 the same need not be true for the volume element. The easiest way to see this surprising fact is to construct orthonormal frames on both $M^{ \pm}$, and compare them along $\Sigma$. We assume that $\Sigma$ is spacelike as seen from both sides, in which case an orthonormal frame on $\Sigma$ can be (separately) extended to orthonormal frames on $M^{ \pm}$by adding the appropriate normal vector, which is spacelike in one case but timelike in the other. However, this discontinuity lies in the metric; the resulting normal vectors, taken together, form a continuous vector field. Since the volume element is just the (wedge) product of the (dual) frame elements, it, too, must be continuous. As mentioned above (and discussed in more detail in [1]), it is enough for the pullbacks of the metric from $M^{ \pm}$to $\Sigma$ to agree in order for there to be a well-defined tangent space on $M$, a condition which is satisfied by this construction.

As discussed in [22], Israel's results [23] relating the stress-energy tensor to the intrinsic and extrinsic curvature of the boundary layer $\Sigma$ must be modified in the presence of signature change. For example, the "energy" density on $\Sigma \subset M^{ \pm}$is now

\footnotetext{
${ }^{1}$ We assume the metrics $g_{a b}^{ \pm}$are non-degenerate on $\Sigma$, the only other possibility.
} 
given by

$$
\rho:=G_{a b} n^{a} n^{b}=\frac{1}{2}\left(\left(K_{c}^{c}\right)^{2}-K_{a b} K^{a b}-\varepsilon R\right)
$$

where $K_{a b}$ is the extrinsic curvature of $\Sigma, R$ is the scalar curvature of $\Sigma$, and $\varepsilon=$ $n_{a} n^{a}= \pm 1$. Imposing Darmois boundary conditions, the curvatures themselves are continuous - but $\varepsilon$ is not. Thus, rather than the Israel condition $[\rho]=0$, we obtain

$$
[\rho]=\left[G_{a b} n^{a} n^{b}\right]=-R .
$$

Furthermore, we can independently recover the extrinsic curvature term from

$$
\left[G^{a}{ }_{b} n^{b} l_{a}\right]=\left(K_{c}^{c}\right)^{2}-K_{a b} K^{a b}
$$

where $l_{a}$ is the dual vector satisfying $l_{a} n^{a}=1$ (on both sides), as using $l_{a}$ instead of $n_{a}$ is equivalent to adding a factor of $\varepsilon$ inside the square brackets. How to interpret "energy" inside a spacelike region is, of course, an open question.

\section{Lorentzian Vector Calculus}

A differential geometer regards vector calculus as "really" being about differential forms. For the last 20 years, in addition to my traditional research in relativity, I have had the pleasure of attempting to implement an approach to the teaching of vector calculus which can be described as "differential forms without differential forms" [12, 13]. Key to this description is the use of the infinitesimal displacement vector $d \vec{r}$, which is really a vector-valued differential form. 2

When moving from Euclidean geometry to Riemannian geometry to Lorentzian geometry, vector calculus as expressed in terms of differential forms is virtually unchanged. However, the conversion to traditional vector language does depend on the signature. It's easy to rewrite the dot product in Lorentzian signature; relativists do this routinely when working with Lorentzian metrics. However, it's less obvious whether there is a cross product, or what it looks like. But nowhere is this dependence on signature more apparent than in the statement of the Divergence Theorem. What is an "outward-pointing" vector field, anyway?

In order to make the analogy to vector calculus more apparent, let's work in $2+1$ dimensional Minkowski space, with orthonormal basis vectors $\hat{x}, \hat{y}$, and $\hat{t}$. This basis satisfies

$$
\hat{x} \cdot \hat{x}=1=\hat{y} \cdot \hat{y}, \quad \hat{t} \cdot \hat{t}=-1,
$$

with all cross terms vanishing. So consider a vector field

$$
\vec{F}=F^{x} \hat{x}+F^{y} \hat{y}+F^{t} \hat{t} .
$$

\footnotetext{
${ }^{2}$ My recent textbook on general relativity [5] also uses this language.
} 
In Minkowski space, the divergence of $\vec{F}$ is

$$
\vec{\nabla} \cdot \vec{F}=\frac{\partial F^{x}}{\partial x}+\frac{\partial F^{y}}{\partial y}+\frac{\partial F^{t}}{\partial t}
$$

there are no minus signs. As in the component-based proof of the (ordinary) Divergence Theorem, we can integrate the divergence over a rectangular box $W$. The first term yields

$$
\iiint_{W} \frac{\partial F^{x}}{\partial x} d x d y d t=\iint \Delta F^{x} d y d t=\int_{S_{x}} \vec{F} \cdot \hat{n} d A
$$

where $S_{x}$ consists of the two faces of the box with outward-pointing normal vectors $\hat{n}= \pm \hat{x}$. A similar expression holds for the $y$-component, but the last equality fails for the $t$ component, since the dot product has the wrong sign. To fix this problem, we must instead choose $\hat{n}$ to be the inward-pointing normal vector on $S_{t}$.

Why this asymmetry? Stokes' Theorem (15) is really about differential forms, and the 1 -form physically equivalent to $\hat{n}$ (with components $n_{a}$ ) is outward-pointing on all of $S$. The "asymmetry" arises due to the metric when converting from 1-forms to vectors.

Why haven't we noticed this asymmetry in relativity? In practice, the Divergence Theorem is not applied to closed regions $W$, but rather to infinite "sandwiches", the region between two spacelike hypersurfaces. The integrals over the timelike sides of the box are replaced by falloff conditions at spatial infinity, leaving only the " $S_{t}$ " contribution in the above argument. The relative sign difference on spacelike and timelike boundaries becomes an overall sign, which can be-and is - safely ignored.

The computations in this section are straightforward, but at first sight the conclusion may be uncomfortable to some readers. The Lorentzian Divergence Theorem does not, in general, involve the outward-pointing normal vector (but rather the outward-pointing 1-form). It is precisely this sort of confrontation between expectation and reality that leads students to an enhanced understanding of the underlying mathematics even in the traditional setting.

The investigation of the Divergence Theorem for regions in Minkowski space whose boundaries contain null pieces is left as an exercise for the reader.

\section{Summary}

We have briefly summarized two quite different bodies of work, the analysis of shells of matter in black-hole spacetimes, and of signature-changing spacetimes, emphasizing the common thread provided by the Patchwork Divergence Theorem, which also sheds new insight on topics in vector calculus. One never knows where the journey will lead.

Thank you, Paddy, for your contributions to my journey. 


\section{Acknowledgments}

It is a pleasure to thank the many collaborators who contributed to my work on piecewise smooth manifolds, including especially Chris Clarke, George Ellis, Charles Hellaby, Gerard 't Hooft, Corinne Manogue, and Robin Tucker.

\section{References}

1. C J S Clarke and Tevian Dray, Junction Conditions for Null Hypersurfaces, Class. Quantum Grav. 4, 265-275 (1987).

2. Tevian Dray, Bouncing Shells, Class. Quantum Grav. 7, L131-L134 (1990)

3. Tevian Dray, Einstein's Equations in the Presence of Signature Change, J. Math. Phys. 37, 5627-5636 (1996).

4. Tevian Dray, Tensor Distributions in the Presence of Degenerate Metrics, Int. J. Mod. Phys. D6, 717-740 (1997).

5. Tevian Dray, Differential Forms and the Geometry of General Relativity, A K Peters/CRC Press, Boca Raton, FL, 2014.

6. Tevian Dray, George Ellis, Charles Hellaby, and Corinne A. Manogue, Gravity and Signature Change, Gen. Rel. Grav. 29, 591-597 (1997).

7. Tevian Dray and Charles Hellaby, The Patchwork Divergence Theorem, J. Math. Phys. 35, 5922-5929 (1994).

8. Tevian Dray and Gerard 't Hooft, The Gravitational Shock Wave of a Massless Particle, Nucl. Phys. B253, 173-188 (1985).

9. Tevian Dray and Gerard 't Hooft, The Effect of Spherical Shells of Matter on the Schwarzschild Black Hole, Commun. Math. Phys. 99, 613-625 (1985).

10. Tevian Dray and Gerard 't Hooft, The Gravitational Effect of Colliding Planar Shells of Matter, Class. Quantum Grav. 3, 825-840 (1986).

11. Tevian Dray and Pankaj Joshi, Glueing Reissner-Nordstrøm Spacetimes Together Along Charged Shells of Matter, Class. Quantum Grav. 7, 41-49 (1990).

12. Tevian Dray and Corinne A. Manogue, Bridging the Vector Calculus Gap (website), 20002016, http: //math.oregonstate.edu/bridge Cited 24 Jun 2016.

13. Tevian Dray and Corinne A. Manogue, The Geometry of Vector Calculus, 2009-2016, http: //math.oregonstate.edu/BridgeBook Cited 24 Jun 2016.

14. Tevian Dray, Corinne A. Manogue, and Robin W. Tucker, Particle Production from Signature Change, Gen. Rel. Grav. 23, 967-971 (1991).

15. Tevian Dray, Corinne A. Manogue, and Robin W. Tucker, The Scalar Field Equation in the Presence of Signature Change, Phys. Rev. D48, 2587-2590 (1993).

16. Tevian Dray, Corinne A. Manogue, and Robin W. Tucker, Boundary Conditions for the Scalar Field in the Presence of Signature Change, Class. Quantum Grav. 12, 2767-2777 (1995).

17. Tevian Dray and T. Padmanabhan, Conserved Quantities from Piecewise Killing Vectors, Gen. Rel. Grav. 21, 741-745 (1989).

18. G. F. R. Ellis, Covariant change of signature in classical relativity, Gen. Rel. Grav. 24, 10471068 (1992)

19. G. Ellis, A. Sumeruk, D. Coule, C. Hellaby, Change of signature in classical relativity, Class. Quantum Grav. 9, 1535-1554 (1992).

20. David Hartley, Robin W. Tucker, Philip Tuckey, and Tevian Dray, Tensor Distributions on Signature-Changing Space-Times, Gen. Rel. Grav. 32, 491-504 (2000).

21. Jeffrey S. Hazboun and Tevian Dray, The Effect of Negative-Energy Shells on the Schwarzschild Black Hole, Gen. Rel. Grav. 42, 1457-1467 (2010).

22. Charles Hellaby and Tevian Dray, Failure of Standard Conservation Laws at a Classical Change of Signature, Phys. Rev. D49, 5096-5104 (1994).

23. Israel, W., Singular hypersurfaces and thin shells in general relativity, Nuov. Cim. 44B, 1-14 (1966). 